\title{
Seleção de isolados do fungo entomopatogênico Beauveria bassiana (Ascomycetes: Clavicipitaceae) para o controle de Rhipicephalus (Boophilus) microplus (Acari: Ixodidae)
}

\author{
Selection of isolates of entomopathogenic fungus Beauveria bassiana (Ascomycetes: Clavicipitaceae)
} for control of Rhipicephalus (Boophilus) microplus (Acari: Ixodidae)

Leila A. G. Barci ${ }^{1 *}$; José Eduardo M. de Almeida²; Adriana H. de Campos Nogueira3; Luciano O. Zappelini²; Angelo P. do Prado ${ }^{4}$

\author{
${ }^{1}$ Instituto Biológico, Centro de Pesquisa e Desenvolvimento de Sanidade Animal - CPDSA \\ ${ }^{2}$ Instituto Biológico, Centro Experimental do Instituto Biológico - CEIB \\ ${ }^{3}$ Apta Regional, Polo Extremo Oeste \\ ${ }^{4}$ Departamento de Parasitologia, Universidade Estadual de Campinas - UNICAMP
}

Recebido em 2 de Outubro de 2008

Aceito em 15 de Setembro de 2009

\section{Resumo}

O presente estudo foi conduzido com o objetivo de selecionar isolados do fungo entomopatogênico Beauveria bassiana com potencial patogênico para utilização no controle do carrapato Rhipicephalus (Boophilus) microplus. Numa primeira etapa, foi avaliada a eficiência de trinta isolados na concentraçáo de $5 \times 10^{8}$ conídios. $\mathrm{mL}^{-1}$. Destes, oito (IBCB01, IBCB02, IBCB07, IBCB17, IBCB21, IBCB74, IBCB149, IBCB165) apresentaram eficiência entre 90 e 99\%; treze (IBCB03, IBCB14, IBCB16, IBCB24, IBCB95, IBCB97, IBCB102, IBCB141, IBCB146, IBCB147, IBCB150, IBCB154, IBCB157), indicaram percentuais de eficiência entre 80 e 89,5\%; seis (IBCB47, IBCB75, IBCB84, IBCB145, IBCB161, IBCB164), entre 70 e 79\% e apenas dois, (IBCB13 e IBCB143) revelaram-se pouco virulentos com porcentagens abaixo de $70 \%$. Na segunda etapa do trabalho, foram analisados comparativamente dados de mortalidade acumulada dos cinco melhores isolados obtidos na primeira fase (IBCB01, IBCB07, IBCB21, IBCB66, IBCB165). Analisando-se os resultados obtidos in vitro, pode-se imputar que os isolados IBCB21 e IBCB66 são os que apresentam maior potencial para utilização a campo, tendo em vista o controle de $R$. (B.) microplus. Os isolados selecionados, na primeira fase, também foram testados quanto à potencialidade de produção massal em meio de arroz pré-cozido. A melhor produção massal foi obtida com o isolado IBCB66.

Palavras-chave: Carrapato, controle biológico, bovinos.

\begin{abstract}
This study was carried out to select isolates of the entomopathogenic fungus Beauveria bassiana with pathogenic potential to control the Rhipicephalus (Boophilus) microplus tick. The effectiveness of thirty isolates was first tested at a concentration of $5 \times 10^{8}$ conidia. $\mathrm{mL}^{-1}$. Of these, eight were evaluated (IBCB01, IBCB02, IBCB07, IBCB17, IBCB21, IBCB74, IBCB149, IBCB165) and showed an effectiveness between 90 and 99\%; thirteen (IBCB03, IBCB14, IBCB16, IBCB24, IBCB95, IBCB97, IBCB102, IBCB141, IBCB146, IBCB147, IBCB150, IBCB154, IBCB157) between 80 and 89,5\%; six (IBCB47, IBCB75, IBCB84, IBCB145, IBCB161, IBCB164) between 70 and $79 \%$, and only two (IBCB13 and IBCB143) had lower pathogenicity (70\% or below). In the second step of the study, the five more effective strains in the first phase of the experiment (IBCB01, IBCB07, IBCB21, IBCB66, IBCB165) were analyzed comparatively. Based on in vitro results, it can be concluded that IBCB66 and IBCB21 are the isolates with higher potential for field control of $R$. (B.) microplus. IBCB01, IBCB07, IBCB21, IBCB66 e IBCB165 isolates were submitted to a conidial production test using a rice-based substrate. The best mass production of the entomopathogenic fungus was obtained with the IBCB66 strain.
\end{abstract}

Keywords: Tick, biological control, cattle.

\footnotetext{
*Autor para correspondência: Leila Aparecida Gardiman Barci

Centro de Pesquisa e Desenvolvimento de Sanidade Animal - CPDSA,

Instituto Biológico, Av. Conselheiro Rodrigues Alves 1.252,

CEP 04.014-002, São Paulo - SP, Brasil

e-mail: barci@biologico.sp.gov.br
} 


\section{Introdução}

O carrapato Rhipicephalus (Boophilus) microplus é um dos principais ectoparasitas de bovinos dos rebanhos tropicais e subtropicais. Causa consideráveis perdas econômicas relacionadas ao próprio parasitismo, transmissão de agentes patogênicos, redução na produção de leite, carne e couro e altos custos com produtos destinados ao seu combate e às doenças por ele transmitidas (HORN; ARTECHE, 1985).

Atualmente, o controle do carrapato é baseado, principalmente, no emprego de produtos químicos aplicados com o objetivo de eliminar as fases parasitárias que infestam os animais. A má utilização dos produtos, associada à questão da resistência, vem aumentando em larga escala os problemas relacionados a esses ectoparasitas. Os efeitos gerados por essa situação estão associados ao crescente uso de diferentes carrapaticidas químicos e ao esgotamento rápido de moléculas que demandam altos custos e necessitam de muito tempo para serem desenvolvidas antes da comercialização. Outras questôes, não menos sérias, estão relacionadas à contaminação do homem, do meio ambiente e dos resíduos nos produtos a serem comercializados (FRANCO, 2000). Considerando-se o mundo globalizado, cujo mercado é cada vez mais competitivo e exigente em termos de certificação de qualidade, esses fatores são entraves determinantes quando se pretende atribuir índices mais altos de valor agregado aos produtos.

Entre as opçóes de controle alternativo de carrapato, destaca-se o emprego do controle microbiano que, na área agrícola, já vem sendo utilizado há muito tempo, individualmente ou em programas de manejo integrado de pragas (ALMEIDA, 1994; ALMEIDA et al. 1997; BATISTA FILHO et al., 1998; TAMAI et al., 2002; MOINO JUNIOR et al., 2002).

Publicaçóes veiculadas na literatura nacional e internacional apontam os fungos entomopatogênicos como os mais promissores para utilização como método de controle do carrapato $R$. (B.) microplus, sendo as espécies Metarhizium anisopliae e Beauveria bassiana as mais pesquisadas (BARCI, 1997; SAMISH et al., 2004).

Estudos desenvolvidos com diversas estirpes de fungos mostraram que a variabilidade genética dos isolados, principalmente quanto a parâmetros moleculares e de patogenicidade, determinam diferenças no grau de virulência do patógeno, interferindo, dessa maneira, nos percentuais de eficiência de controle de insetos e ácaros (ALMEIDA et al., 1997; TAMAI et al., 2002; FERNANDES et al., 2006).

Dados divulgados em periódicos nacionais apontam diferentes índices de eficiência de isolados de Metarhizium anisopliae para ovos, larvas e fêmeas de carrapatos de bovinos (BITTENCOURT, 1992; BITTENCOURT et al., 1992, 1994a, 1994b, 1995b).

Em relação à B. bassiana, Bittencourt et al. (1995a, 1996, 1997a, 1997b) relataram, em condiçóes de laboratório, a ação patogênica de diferentes isolados dessa espécie de fungo nos estágios de vida livre de $R$. (B.) microplus. Descreveram alteraçóes nos períodos de pré-postura, postura, eclosão, percentual de eclosão, índice de eficiência reprodutiva e nutricional nas diversas etapas da fase não parasitária de carrapatos após terem sido submetidas a tratamentos com diferentes concentraçóes de conídios do fungo $B$. bassiana.

Fernandes et al. (2006) estudaram a patogenicidade de isolados de $B$. bassiana para larvas não alimentadas de $R$. (B.) microplus.
Relacionaram o efeito deletério produzido nesses instares à variabilidade genética das estirpes e constataram que alguns isolados, apesar de patogênicos, não são eficazes, pois, mesmo quando utilizados em altas concentraçóes, não produziram índices de mortalidade satisfatórios.

Dessa maneira, é primordial que se desenvolvam pesquisas direcionadas à seleção de isolados para que se conheça a potencialidade dos diferentes materiais genéticos e se possa eleger o(s) mais adequado(s) para sua utilização em programas de controle biológico de carrapatos. Levando-se em conta que a coleção do Instituto Biológico conta com grande número de isolados os quais são amplamente utilizados no combate de pragas agrícolas (ALMEIDA et al., 1997; ALMEIDA et al., 1998; MOINO JUNIOR et al., 2002; TAMAI et al., 2002), o presente trabalho teve por objetivo avaliar in vitro a suscetibilidade de larvas não alimentadas de $R$. (B.) microplus à ação patogênica de 30 isolados de $B$. bassiana.

\section{Material e Métodos}

Os testes experimentais foram conduzidos no Instituto Biológico nos laboratórios de Parasitologia Animal (Centro de Pesquisa e Desenvolvimento de Sanidade Animal) e Controle Biológico (Centro Experimental do Instituto Biológico).

Fêmeas ingurgitadas foram coletadas diretamente do animal infestado experimentalmente, levadas ao laboratório, onde foram separadas e identificadas quanto ao gênero e espécie, segundo critérios preconizados pela chave de Aragão e Fonseca (1961), lavadas com água destilada, secas em papel macio, colocadas em placas de Petri e mantidas em estufa BOD sob $26,5{ }^{\circ} \mathrm{C}$ de temperatura e $80 \%$ de umidade relativa. Quinze dias após o início da oviposição, as posturas foram separadas em alíquotas de $500 \mathrm{mg}$ e colocadas em frascos de vidro com capacidade para $3 \mathrm{~mL}$ fechados com tecido de organza. Os ovos e as larvas deles eclodidas foram mantidas sob as mesmas condiçóes, em estufa BOD. Nos bioensaios, foram utilizadas larvas infestantes com idade entre 15 e 18 dias.

Foram avaliados 30 isolados de $B$. bassiana os quais se encontram depositados no Banco de Microrganismos Entomopatogênicos "Oldemar Cardim Abreu" do Laboratório de Controle Biológico/ Centro Experimental do Instituto Biológico.

As suspensóes de conídios foram preparadas a partir dos fungos removidos do meio batata-dextrose-agar (BDA), diluídos em água destilada acrescida de Tween $80^{\circledR} 0,1 \%$ e quantificados por meio de contagem em câmara de Neubauer (ALVES; MORAES, 1998). A partir da suspensão-mãe, foram preparadas suspensões dos diferentes isolados na concentração de $5 \times 10^{8}$ conídios. $\mathrm{mL}^{-1}$, de acordo com a CL90 definida para o isolado padrão IBCB66.

O experimento foi desenvolvido em duas fases:

- Fase 1: Nesse estágio, os isolados foram avaliados somente quanto à eficiência. Os trinta isolados foram divididos em grupos de cinco isolados mais o padrão e testados na concentração de $5 \times 10^{8}$ conídios. $\mathrm{mL}^{-1}$. O grupo testemunha recebeu como tratamento água destilada com espalhante adesivo Tween $80^{\circledR}$. A avaliação do ensaio foi realizada 16 dias após o início do bioensaio. 
- Fase 2: Após determinar a eficiência dos 29 isolados, juntamente com o padrão IBCB66, os que apresentaram percentual de mortalidade superior a $95 \%$ foram novamente testados.

Nessa fase, as leituras foram feitas diariamente com o objetivo de se conhecer a distribuição da mortalidade acumulada.

Para o desenvolvimento do experimento foi utilizada a metodologia descrita por Barci e Nogueira (2006), que permite a observação diária de indivíduos de um mesmo tratamento, pelo menos por 20 dias, sem que haja alteração significativa nos percentuais de mortalidade do grupo controle.

O delineamento experimental utilizado foi o inteiramente casualizado, constando de 10 repetiçóes por tratamento, sendo utilizadas 20 larvas em cada repetição, perfazendo um total de 200 indivíduos por tratamento. Os resultados obtidos na fase $1 \mathrm{e}$ 2 foram analisados utilizando o teste ANOVA. As médias foram comparadas pelo teste de comparação múltipla de Tukey a 5\% de significância. A mortalidade corrigida foi calculada segundo fórmula descrita por Abbott (1925).

Procedimentos referentes à produção massal de fungos em meio de arroz cozido foram conduzidos no Laboratório de Controle Biológico do Centro Experimental do Instituto Biológico, situado em Campinas, SP. Os isolados dos fungos B. bassiana IBCB01, IBCB07, IBCB21, IBCB66 e IBCB165 foram produzidos conforme descrição de Leite et al. (2003).

Para a quantificação dos conídios, foi retirado $1 \mathrm{~g}$ do arroz produzido de cada isolado, o qual foi diluído em $10 \mathrm{~mL}$ de suspensão autoclavada de água destilada e Tween $80^{\circ}$. Os frascos contendo as suspensóes foram agitados e diluídos cerca de 1.000 vezes para a retirada de uma pequena alíquota, suficiente para contagem em câmara de Neubauer.

Os bioensaios foram realizados utilizando-se cinco repetições para cada isolado.

Os valores consignados foram transformados em raiz quadrada $(\mathrm{x}+0,5)$ e submetidos à análise de variância, utilizando-se o teste de Tukey $(\alpha=0,05)$ para comparar as médias de produção de conídios.

\section{Resultados}

Fase 1: As porcentagens de mortalidade de larvas de $R$. (B.) microplus tratadas com os $5 \times 10^{8}$ conídios. $\mathrm{mL}^{-1}$ dos isolados de $B$. bassiana testados encontram-se reunidas na Tabela 1 .

Dos trinta isolados testados, sete $(23,33 \%)$ apresentaram percentuais de eficiência iguais ou superiores a $90 \%$; treze $(43,33)$ apontaram índices de eficiência iguais ou superiores a 80 e inferiores a $90 \%$; oito $(23,3 \%)$ registraram percentuais iguais ou superiores a 70 e inferiores a $80 \%$. Em apenas um isolado $(3,3 \%)$ foi registrado percentual de mortalidade entre 60 e 70\%; assim como em um isolado foi constatado índice de mortalidade entre 20 e 40\%. Nenhum isolado registrou percentuais de mortalidade inferiores a 20\% nem entre a faixa compreendida entre 40 e $60 \%$ (Figura 1).

Dos 20 isolados que apresentaram percentuais de eficiência acima de 81\%, treze: (IBCB03, IBCB14, IBCB24, IBCB66, IBCB95, IBCB97, IBCB102, IBCB141, IBCB146, IBCB147, IBCB150, IBCB154 e IBCB157) estão agrupados na faixa entre

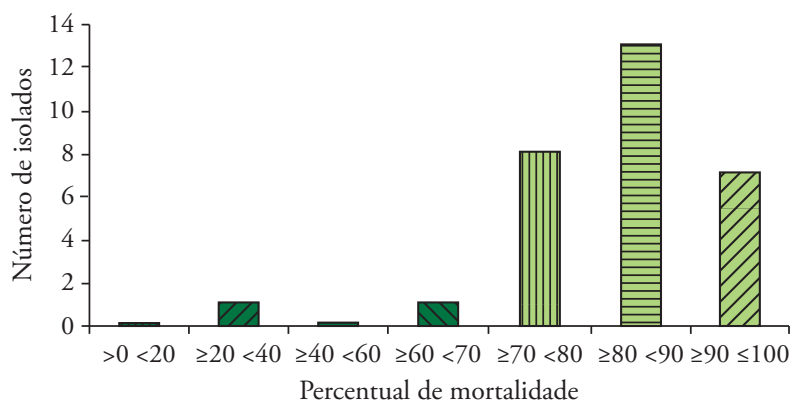

Figura 1. Percentuais de mortalidade obtidos nos tratamentos de larvas de Rhipicephalus (Boophilus) microplus inoculadas com $5 \times 10^{8}$ conídios de 30 isolados de Beauveria bassiana (Fase 1).

Tabela 1. Mortalidade (\%) de larvas de Rhipicephalus (Boophilus) microplus tratadas com suspensóes de $5 \times 10^{8}$ conídios. $\mathrm{mL}^{-1}$ de trinta isolados de Beauveria bassiana (ordem decrescente).

\begin{tabular}{cccc}
\hline Isolado & Mortalidade (\%) & Isolado & Mortalidade (\%) \\
\hline IBCB01 & 98,5 & IBCB14 & 82,0 \\
IBCB21 & 97,0 & IBCB154 & 82,0 \\
IBCB07 & 96,0 & IBCB97 & 81,0 \\
IBCB165 & 95,5 & IBCB146 & 81,0 \\
IBCB17 & 95,0 & IBCB16 & 80,0 \\
IBCB02 & 92,0 & IBCB66 & $80,8 *$ \\
IBCB149 & 92,0 & IBCB75 & 78,0 \\
IBCB03 & 89,5 & IBCB47 & 77,0 \\
IBCB102 & 89,0 & IBCB145 & 77,0 \\
IBCB141 & 89,0 & IBCB84 & 76,0 \\
IBCB95 & 88,0 & IBCB161 & 74,0 \\
IBCB150 & 87,0 & IBCB164 & 73,0 \\
IBCB147 & 86,0 & IBCB74 & 72,0 \\
IBCB24 & 85,0 & IBCB13 & 65,5 \\
IBCB157 & 85,0 & IBCB143 & 33,0 \\
\hline
\end{tabular}

*Média das porcentagens de mortalidade do isolado padrão obtida nos cinco experimentos.

81 e $90 \%$ e sete (IBCB01, IBCB02, IBCB07, IBCB17, IBCB21, IBCB149 e IBCB165) mostraram porcentagens acima de 91\%.

Fase 2: Os resultados obtidos possibilitaram observar que os percentuais de mortalidade de larvas tratadas com $5 \times 10^{8}$ conídios. $\mathrm{mL}^{-1}$ dos isolados IBCB01, IBCB07, IBCB21, IBCB165 e IBCB66 variaram entre 70,5 e 92,5\%. A mortalidade do grupo controle foi de $1,5 \%$.

Os índices de mortalidade acumulada e corrigida foram muito próximos (Figura 2). Os resultados descritos encontram-se agrupados na Tabela 2 .

A produção de conídios dos isolados IBCB01, IBCB07, IBCB21, IBCB66 e IBCB165 de Beauveria bassiana em meio de arroz pré-cozido, estáo representados na Figura 3. O isolado IBCB66 apresentou maior produção que os demais isolados, tendo alcançado quase o dobro da produção do segundo melhor, o IBCB07. Já os isolados IBCB01, IBCB 21 e IBCB165 obtiveram as menores produçóes de conídios por grama de arroz. 


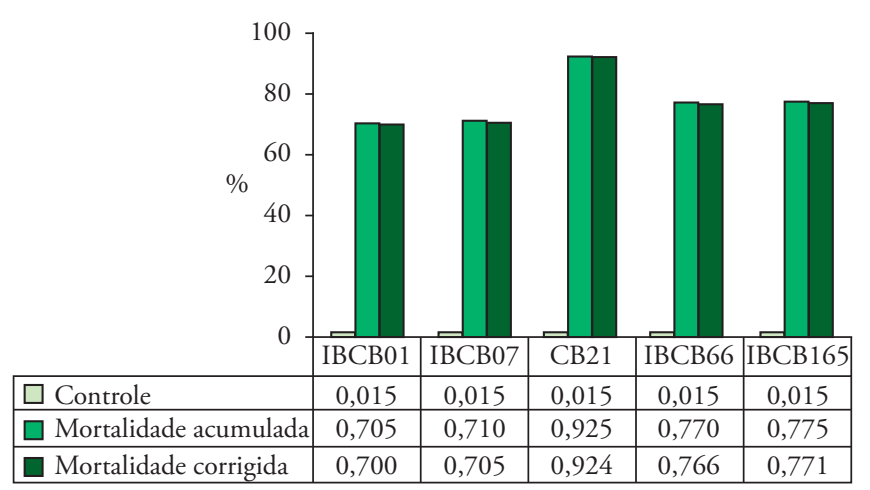

Figura 2. Porcentagem de mortalidade acumulada e corrigida de larvas de Rhipicephalus (Boophilus) microplus inoculadas com suspensão de $5 \times 10^{8}$ conídios. $\mathrm{mL}^{-1}$ dos isolados IBCB01, IBCB07, IBCB66 e IBCB165 de Beauveria bassiana.



Figura 3. Produçáo de conídios dos isolados IBCB01, IBCB07, IBCB21, IBCB66 e IBCB165 de Beauveria bassiana em meio de arroz pré-cozido.

\section{Discussáo}

$\mathrm{Na}$ área agrícola, a seleção de isolados de organismos entomopatogênicos é um procedimento bastante comum, quando se pretende estabelecer estratégias de manejo integrado de pragas as quais consideram o controle microbiano como um elemento do conjunto de etapas a serem implementadas. A utilização de linhagens e/ou isolados apropriados de agentes biológicos é de fundamental importância para o desenvolvimento de um programa de controle microbiano. Para tanto, é imprescindível contar com um bom banco de isolados, devidamente preservado e com variabilidade genética comprovada, para, entâo, iniciar o programa a partir da seleção dos materiais promissores.

A variabilidade genética dos isolados da coleçáo de entomopatógenos do Instituto Biológico já foi comprovada, principalmente, quanto à virulência e patogenicidade para insetos e ácaros pragas (ALMEIDA et al., 1997; TAMAI et al., 2002.), constituindo-se, dessa maneira, em reservatórios diversificados para estudos com enfoque na seleção de materiais que apresentem características adequadas para sua utilização em programas de controle biológico de pragas agrícolas e veterinárias.

Os percentuais de mortalidade obtidos na Fase 1 permitem inferir que os isolados de $B$. bassiana, depositados na Coleção de Entomopatógenos do Instituto Biológico, apresentam capacidade de produzir infecção em larvas de $R$. (B.) microplus e, portanto, têm potencial de utilização em programas de controle do carrapato de bovinos.

Essas observaçóes vão ao encontro dos relatos da literatura nacional que registra excelentes resultados de experimentaçóes conduzidas em condiçóes de laboratório, ao avaliar o efeito de diversas estirpes de $B$. bassiana para larvas de $R$. (B.) microplus (FERNANDES et al., 2006).

As observaçóes realizadas na presente pesquisa estão em concordância com aquelas mostradas por Bittencourt et al. (1996), que estudaram a eficiência de dois isolados de B. bassiana - 986 e 747 - sobre ovos e larvas de $R$. (B.) microplus. Os autores concluíram que os isolados 986 e 747 eram patogênicos para larvas de $R$. (B.) microplus e que se tratava de um inimigo natural com potencial para ser usado no combate ao ectoparasita.

As mesmas afirmaçóes foram reportadas por Paiáo et al. (2001), que, ao pesquisar a suscetibilidade do carrapato bovino frente a isolados de $B$. bassiana, observaram um acréscimo na taxa de mortalidade de larvas tratadas de 0,8 a 70,49\%. Os mesmos autores apontaram os isolados AM09, CB07 e JAB07 como os mais efetivos.

Os dados registrados neste trabalho também corroboram os resultados publicados por Fernandes et al. (2003), que isolaram três estirpes - Bb28, Bb29 e Bb30 de fêmeas ingurgitadas de $R$. (B.) microplus coletadas a campo. Testes realizados em condiçóes de laboratório, conduzidos com os isolados supramencionados, permitiram verificar altas porcentagens de mortalidade de larvas tratadas em comparação ao grupo controle.

Analisando-se os percentuais de eficiência dos isolados de $B$. bassiana testados para larvas de $R$. (B.) microplus, pode-se verificar que, dos trinta isolados avaliados, sete apresentaram índices entre 90 e 99\%, treze indicaram valores entre 80 e $89 \%$, oito entre 70 e $79 \%$ e apenas dois revelaram-se menos patogênicos com porcentagens abaixo de $70 \%$. Com base nesses dados, obtidos em condiçóes de laboratório, pode-se concluir que 93,3\% dos isolados avaliados demonstraram elevada eficiência no controle de larvas de $R$. (B.) microplus. ( $\geq 70 \%)$. Dependendo da formulação utilizada, os 20 isolados que apresentaram índices superiores a $80 \%$, possivelmente, teriam potencial patogênico suficiente para serem utilizados a campo, o que representa uma possibilidade de utilização de 66,6\%.

Ao confrontar os resultados obtidos na Fase 2, que teve por finalidade estabelecer comparação entre os melhores isolados, ressalta-se que o percentual de mortalidade do grupo-controle foi extremamente baixo, resultado que permitiu inferir grande confiabilidade ao bioensaio e estabelecer paralelos comparativos com relativa margem de segurança.

A análise estatística do número de mortos acumulados de larvas de $R$. (B.) microplus submetidas aos diferentes tratamentos permitiu estabelecer uma comparação diária entre os isolados utilizados na experimentação. 
Tabela 2. Número acumulado de larvas de Rhipicephalus (Boophilus) microplus mortas após tratamento com diferentes isolados de Beauveria bassiana no período compreendido entre o $1^{\circ}$ e o $16^{\circ}$ DAÍ.

\begin{tabular}{|c|c|c|c|c|c|c|c|c|}
\hline \multirow{2}{*}{ Tratamento } & \multicolumn{8}{|c|}{ Dias após o tratamento } \\
\hline & 1 & 2 & 3 & 4 & 5 & 6 & 7 & 8 \\
\hline Controle & $0 \pm 0$ & $0 \pm 0$ & $0,1 \pm 0,3162^{a}$ & $0,1 \pm 0,3162^{\mathrm{a}}$ & $0,1 \pm 0,3162^{\mathrm{a}}$ & $0,1 \pm 0,3162^{\mathrm{a}}$ & $0,1 \pm 0,3162^{\mathrm{a}}$ & $0,1 \pm 0,3162^{\mathrm{a}}$ \\
\hline IBCB01 & $0,1 \pm 0,3162^{a}$ & $0,1 \pm 0,3162^{\mathrm{ab}}$ & $0,1 \pm 0,3162^{a}$ & $0,1 \pm 0,3162^{\mathrm{a}}$ & $0,2 \pm 0,4216^{\mathrm{a}}$ & $0,4 \pm 0,5164^{a}$ & $0,5 \pm 0,5270^{\mathrm{a}}$ & $0,8 \pm 0,6325^{a}$ \\
\hline IBCB07 & $0 \pm 0$ & $0 \pm 0$ & $0 \pm 0$ & $0 \pm 0$ & $0 \pm 0$ & $0 \pm 0$ & $0,2 \pm 0,6325^{\mathrm{a}}$ & $0,4 \pm 0,6992^{a}$ \\
\hline IBCB21 & $0,3 \pm 0,4830^{a}$ & $0,7 \pm 0,8233^{a}$ & $1,3 \pm 1,3375^{b}$ & $1,5 \pm 1,4337^{b}$ & $1,7 \pm 1,7670^{b}$ & $2,5 \pm 2,3214^{b}$ & $3,2 \pm 2,7809^{b}$ & $4,1 \pm 3,1073^{b}$ \\
\hline IBCB66 & $0,2 \pm 0,4216^{a}$ & $0,3 \pm 0,4830^{\mathrm{ab}}$ & $0,4 \pm 0,5164^{a}$ & $0,4 \pm 0,5164^{a}$ & $0,4 \pm 0,5164^{\mathrm{a}}$ & $0,6 \pm 0,6992^{a}$ & $0,7 \pm 0,8233^{a}$ & $0,8 \pm 0,7888^{a}$ \\
\hline IBCB165 & $0 \pm 0$ & $0,1 \pm 0,3162^{\mathrm{ab}}$ & $0,1 \pm 0,3162^{\mathrm{a}}$ & $0,1 \pm 0,3162^{a}$ & $0,1 \pm 0,3162^{a}$ & $0,1 \pm 0,3162^{a}$ & $0,4 \pm 0,6992^{a}$ & $0,5 \pm 0,8498^{a}$ \\
\hline $\mathrm{CV}$ & 19,1 & 26,2 & 29,2 & 30,4 & 32,2 & 35,2 & 36,8 & 37,8 \\
\hline $\mathrm{F}$ & 2,04 & 3,6 & 6,9 & 7,7 & 7,1 & 9,6 & 9,2 & 10,5 \\
\hline \multirow{2}{*}{ Tratamento } & \multicolumn{8}{|c|}{ Dias após o tratamento } \\
\hline & 9 & 10 & 11 & 12 & 13 & 14 & 15 & 16 \\
\hline Controle & $0,1 \pm 0,3162^{a}$ & $0,1 \pm 0,3162^{a}$ & $0,2 \pm 0,4216^{a}$ & $0,2 \pm 0,4216^{a}$ & $0,2 \pm 0,4216^{a}$ & $0,2 \pm 0,4216^{a}$ & $0,2 \pm 0,4216^{a}$ & $0,3 \pm 0,6749^{a}$ \\
\hline IBCB01 & $1,2 \pm 0,6325^{a}$ & $1,8 \pm 1,1353^{b}$ & $1,8 \pm 1,1353^{\mathrm{ab}}$ & $2,5 \pm 1,7795^{b}$ & $3,7 \pm 2,9078^{b}$ & $5,2 \pm 3,7357^{b}$ & $7,0 \pm 3,8297^{b}$ & $9,0 \pm 4,0000^{b}$ \\
\hline IBCB07 & $0,7 \pm 0,8233^{a}$ & $1,0 \pm 0,8165^{\mathrm{ab}}$ & $1,5 \pm 0,9718^{\mathrm{ab}}$ & $2,7 \pm 1,3375^{b}$ & $3,9 \pm 1,9120^{b}$ & $5,0 \pm 1,8856^{b}$ & $7,6 \pm 2,2706^{b}$ & $10,0 \pm 3,3665^{b}$ \\
\hline IBCB21 & $5,2 \pm 3,7357^{b}$ & $7,1 \pm 4,9989 \mathrm{c}$ & $8,2 \pm 5,3500$ & $10,2 \pm 5,0067$ & $11,9 \pm 5,0870$ & $13,3 \pm 5,1435$ & $14,3 \pm 4,9453$ & $15,7 \pm 3,5606$ \\
\hline IBCB66 & $1,1 \pm 0,8756^{a}$ & $1,4 \pm 0,9661^{\mathrm{ab}}$ & $1,6 \pm 1,1738^{\mathrm{ab}}$ & $2,1 \pm 1,7288^{b}$ & $3,4 \pm 1,8379^{b}$ & $5,3 \pm 3,1640^{b}$ & $6,2 \pm 3,3928^{b}$ & $8,4 \pm 4,0607^{b}$ \\
\hline IBCB165 & $1,1 \pm 0,9944^{\mathrm{a}}$ & $2,3 \pm 1,6364^{\mathrm{b}}$ & $3,0 \pm 1,4142^{b}$ & $3,9 \pm 1,5239^{b}$ & $6,0 \pm 3,0185^{b}$ & $7,2 \pm 2,6998^{b}$ & $8,6 \pm 2,9889^{b}$ & $10,5 \pm 2,9907^{\mathrm{b}}$ \\
\hline $\mathrm{CV}$ & 36,7 & 34,3 & 31,6 & 27,8 & 27,0 & 25,4 & 20,4 & 17,1 \\
\hline $\mathrm{F}$ & 10,8 & 14,0 & 17,1 & 23,7 & 22,9 & 23,7 & 34,3 & 47,0329 \\
\hline
\end{tabular}

Dados originais seguidos do erro padrão da média; Médias seguidas da mesma letra na coluna não diferem estatisticamente entre si pelo teste de Tukey (5\%); Teste: One - Way ANOVA; Dados transformados em Raiz quadrada $(x+0,5)$ para fins de análise estatística; DAI - Dias após inoculação; CV - coeficiente de variação; e $\mathrm{F}$ - Teste F.

O fato de não haver diferenças estatísticas entre os tratamentos nos dias 1 e 2 era esperado, uma vez que a ação do fungo é fundamentalmente mecânica e, de acordo com Alves (1998), em relação a insetos, o tempo necessário para colonização pode variar de 72 a 120 horas, dependendo do hospedeiro, do patógeno e das condiçốes ambientais.

Os dados apurados entre os dias 3 até 9, sob a ótica da análise estatística, permitiram deduzir que, durante esse período, com exceção do isolado IBCB21, todos os outros tratamentos apresentaram processo de colonização pelo entomopatógeno semelhante, o qual não foi suficientemente deletério para promover percentual de mortalidade capaz de discrepar estatisticamente daquele apresentado nos indivíduos que compuseram o grupo controle.

No $10^{\circ}$ DAI, os isolados IBCB01 e IBCB165 começaram a se diferenciar da testemunha, indicando que, provavelmente, esse seja o tempo necessário para que esses isolados apresentem ação patogênica suficiente para controlar larvas do carrapato sob condiçóes controladas. Entretanto, a mesma observação no 11 DAI foi válida apenas para o isolado IBCB 165.

Os valores observados no período compreendido entre o $12^{\circ} \mathrm{e}$ 160 DAI, segundo análise estatística, permitiram consolidar o possível perfil de comportamento dos isolados no decorrer da experimentação. Todos os grupos tratados apresentaram potencial patogênico suficiente, para induzir percentuais de mortalidade significativamente divergentes em relação aos apurados no grupo controle. A ação mais eficaz foi detectada no grupo que foi submetido ao isolado IBCB21. Os outros isolados náo apresentaram diferenças significativas entre si quanto à ação patogênica para larvas de $R$. (B.) microplus.

A análise estatística dos dados de mortalidade acumulada de larvas de $R$. (B.) microplus tratadas com os isolados IBCB01, IBCB07, IBCB21, IBCB66 e IBCB165 de B. bassiana, apurados no final da experimentação, indicaram que o IBCB21 foi o isolado que mais se destacou durante toda a evoluçáo da experimentação, por apresentar maior percentual de mortalidade nos indivíduos que compuseram as dez parcelas do ensaio e, também, evolução crescente e coerente dos percentuais em pauta. A análise supracitada permitiu inferir que, nessa experimentação, o isolado IBCB21 foi o que apresentou melhor desempenho sobre larvas não alimentadas do carrapato $R$. (B.) microplus.

Os valores dos coeficientes de variação $(\mathrm{CV})$ foram mais altos na fase intermediária em razão dos baixos valores das médias dos tratamentos, principalmente nas fases iniciais do experimento.

Analisando-se os dados publicados por Tamai et al. (2002), que selecionaram isolados de $B$. bassiana procedentes do mesmo banco de patógenos para o controle do Tetranichus urticae (ácaro rajado), pode-se verificar que não houve concordância nas duas pesquisas quanto à conclusão referente à eleição dos melhores isolados. Essa constatação, provavelmente, pode ser explicada com base nas diferenças inerentes às peculiaridades anatomofisiológicas entre ácaros fitófagos e hematófagos.

A demonstração maior, revelada pelos resultados obtidos nas duas pesquisas, é que, na Coleção de Entomopatógenos do Instituto 
Biológico, estão depositados isolados de Beauveria bassiana de grande potencial de utilização como controladores de pragas que acometem culturas vegetais e explorações animais.

Entre as qualidades desejadas para um fungo entomopatogênico, Alves (1998) destaca as seguintes: alta eficiência no controle, grande capacidade de disseminação, resistência às condiçóes adversas, além de qualidades industriais como ótima produção de conídios e taxa de crescimento elevada.

Em relação aos dados referentes à produção massal de conídios em arroz pré-cozido, o isolado IBCB66 foi o que apresentou melhor desempenho nessa análise, seguido pelo IBCB07.

Os isolados IBCB01, IBCB21 e IBCB165 não diferiram estatisticamente entre si quanto ao sistema de produção em larga escala.

Levando-se em conta que todas as condiçôes disponíveis para o crescimento dos fungos na massa de arroz foram as mesmas, tais como, entre outras: teor de umidade, tempo de cozimento do arroz, flutuação da temperatura na sala de produçáo e exposição ao mesmo nível de contaminação ao analisar os dados obtidos, pode-se inferir que todos os isolados testados apresentaram níveis de produção de conídios muito próximos.

Testes adicionais são necessários para se avaliar o potencial de cada um desses isolados, visando a sua utilização como formulaçóes micoacaricidas.

\section{Conclusóes}

Todos os isolados testados são eficientes e capazes de promover infecção em larvas de $R$. (B.) microplus mantidas em condiçôes controladas de temperatura e umidade $\left(27^{\circ} \mathrm{C} \pm 1 \mathrm{e}\right.$ $\mathrm{UR} \geq 80 \%)$.

A maioria das cepas de Beauveria bassiana testadas (73\%) tem potencial para utilização no controle de larvas do carrapato R. (B.) microplus.

Os isolados IBCB01, IBCB07, IBCB21, IBCB47, IBCB66 e IBCB165 de B. bassiana são altamente patogênicos para a fase de larva náo alimentada do carrapato $B$. microplus.

O isolado IBCB 21 é o mais virulento e apresentou melhor desempenho quanto à evolução de colonização de larvas não alimentadas do carrapato $R$. (B.) microplus.

O isolado IBCB66 apresentou melhores índices de produção massal em meio de arroz pré-cozido.

\section{Referências}

ABBOTT, W. S. A method for computing the effectiviness of insecticides. Journal of Economic Entomology, v. 18, n. 15, p. 265-267, 1925.

ALMEIDA, J. E. M. Avaliaçáo de fungos entomopatogênicos visando ao controle do cupim subterrâneo Heterotermes tenuis (Hagen, 1858) (Isoptera, Rhinotermitidae). Piracicaba, 1994. 105 p. Dissertação (Mestrado em Entomologia) - Universidade de Sáo Paulo - USP.

ALMEIDA, J. E. M.; ALVES, S. B.; PEREIRA, R. M. Selection of Beauveria spp. isolates for control of the termite Heterotermes tenuis (Hagen, 1858). Journal of Applied Entomology, v. 121, n. 9/10, p. $539-543,1997$.
ALMEIDA, J. E. M. et al. Controle do cupim subterrâneo Heterotermes tenuis (Hagen) com iscas Termitrap impregnadas com inseticidas associados ao fungo entomopatogênico Beauveria bassiana (Bals.) Vuill. Anais da Sociedade Entomológica do Brasil, v. 27, n. 4, p. 639-644, 1998.

ALVES, S. B. Fungos entomopatogênicos. In: ALVES, S. B. (Ed.) Controle microbiano de insetos. 2 ed. Piracicaba: FEALQ, 1998. p. 289-382.

ALVES, S. B.; MORAES, S. A. Quantificação de inóculo de patógenos de insetos. In: ALVES, S.B. (Ed.). Controle microbiano de insetos. 2 ed. Piracicaba: FEALQ, 1998. p. 765-777.

ARAGÃO, H.; FONSECA, F. Notas de Ixodologia: VIII lista e chave para os representantes da fauna ixodológica brasileira. Memórias do Instituto Oswaldo Cruz, v. 59, n. 2, p. 115-129, 1961.

BARCI, L. A. G. Controle biológico do carrapato dos bovinos Boophilus microplus (Acari, Ixodidae) no Brasil. Arquivos do Instituto Biológico, v. 64, n. 1, p. 95-101, 1997.

BARCI, L. A. G.; NOGUEIRA, A. H. C. Método para avaliação de mortalidade de larvas de Boophilus microplus (Canestrini, 1887) submetidas a tratamentos com produtos carrapaticidas. Arquivos do Instituto Biológico, v. 73, n. 1, p. 105-109, 2006

BATISTA-FILHO, A. et al. Formulação de entomopatógenos, In: ALVES, S. B. (Ed.) Controle microbiano de insetos. 2 ed. Piracicaba: FEALQ, 1998. p. 917-965.

BITTENCOURT, V. R. E. P. Açáo do fungo Metarhizium anisopliae (Metchnikoff, 1879) Sorokin, 1883, sobre o carrapato Boophilus microplus (Canestrini, 1887). Rio de Janeiro, 1992. 105 p. Tese (Doutorado em Medicina Veterinária) - Universidade Federal Rural do Rio de Janeiro, UFRRJ.

BITTENCOURT, V. R. E. P.; LIMA, A. F.; MASSARD, C. L. Uso de Metarhizium anisopliae (Metschnikoff,1879) Sorokin, 1883, no controle do carrapato Boophilus microplus (Canestrini,1887). Arquivos da Universidade Federal Rural do Rio de Janeiro, v. 15, n. 2, p. 197-202, 1992.

BITTENCOURT, V. R. E. P.; MASSARD, C. L.; LIMA, A. F. Ação do fungo Metarhizium anisopliae sobre a fase não parasitária do ciclo biológico de Boophilus microplus. Revista Universidade Rural, v. 16, n. 1/2, p. 49-55, 1994a. (Série Ciências da Vida).

BITTENCOURT, V. R. E. P.; MASSARD, C. L.; LIMA, A. F. Ação do fungo Metarhizium anisopliae (Metschnikoff,1879) Sorokin, 1883, em ovos e larvas do carrapato Boophilus microplus (Canestrini,1887). Revista Universidade Rural, v. 16, n. 1/2, p. 41-47, 1994b. (Série Ciências da Vida).

BITTENCOURT, V. R. E. P.; PERALVA, S. L. F. S.; VIEGAS, E. C. Eficácia in vitro dos isolados 747 e 986 do fungo Beauveria bassiana no carrapato Boophilus microplus. Revista Brasileira de Parasitologia Veterinaria, v. 4, n. 2, p. 86, 1995a. (suplemento 1).

BITTENCOURT, V. R. E. P.; MASSARD, C. L.; LIMA, A. F. Dinâmica da infecção do fungo Metarhizium anisopliae (Metschnikoff, 1879) Sorokin, 1883, sobre o carrapato Boophilus microplus (Canestrini, 1887). Revista Universidade Rural, v. 17, n. 1, p. 83-88, 1995b. (Série Ciências da Vida).

BITTENCOURT, V. R. E. P. et al. Avaliação do efeito do contato de Beauveria bassiana (Bals.) Vuill. com ovos e larvas de Boophilus microplus (Canestrini, 1887) (Acari: Ixodidae). Revista Brasileira de Parasitologia Veterinária, v. 5, n. 2, p. 81-84, 1996. 
BITTENCOURT, V. R. E. P. et al. Avaliação da eficácia in vitro de dois isolados do fungo entomopatogênico Beauveria bassiana (Bals.) Vuill. em fêmeas ingurgitadas de Boophilus microplus (Canestrini, 1887) (Acari: Ixodidae). Revista Brasileira de Parasitologia Veterinária, v. 6, n. 1, p. 49-52, 1997a.

BITTENCOURT, V. R. E. P. et al. Açáo in vitro de dois isolados do fungo entomopatogênico Beauveria bassiana em alguns parâmetros da fase não parasitária de fêmeas ingurgitadas de Boophilus microplus. Revista Universidade Rural, v. 19, n. 1/2, p. 65-71, 1997b. (Série Ciências da Vida).

FERNANDES, E. K. et al. Beauveria bassiana isolated from engorged females and tested against eggs and larvae of Boophilus microplus (Acari: Ixodidae). Journal of Basic Microbiology, v. 43, n. 5, p. 393-398, 2003.

FERNANDES, E. K. K. et al. Study on morphology, pathogenicity, and genetic variability of Beauveria bassiana isolates obtained from Boophilus microplus tick. Parasitology Research, v. 98, n. 4, p. 324-332, 2006.

FRANCO, M. Vem aí a vacina contra o carrapato. DBO Rural, v. 19, n. 235 , p.114-116, 2000
HORN, S. C.; ARTECHE, C. C. P. Situação parasitária da pecuária no Brasil. Hora Veterinária, v. 4, n. 23, p. 12-32, 1985.

LEITE, L. G. et al. Produção de Fungos Entomopatogênicos. Ribeirão Preto: A. S. Pinto, 2003. p. 59.

MOINO JUNIOR., A. et al. External development of the entomopathogenic fungi Beauveria bassiana and Metarhizium anisopliae in the subterranean termite Heterotermes tenuis. Scientia Agricola, v. 59, n. 2, p. 267-273, 2002.

PAIÁO, J. C. V.; MONTEIRO, A. C.; KRONKA, S. N. Susceptibility of the cattle tick Boophilus microplus (Acari: Ixodidae) to isolates of the fungus Beauveria bassiana. World Journal of Microbiology and Biotechnology, v. 17, n. 3, p. 245-251, 2001.

SAMISH, M.; GINSBERG, H.; GLAZER, I. Biological control of ticks. Parasitology, v. 129, p. 389-403, 2004. (suplemento).

TAMAI, M. A. et al. Avaliação de fungos entomopatogênicos para o controle de Tetranychus urticae Koch (Acari: Tetranychidae). Arquivos do Instituto Biológico, v. 69, n. 3, p. 77-84, 2002. 